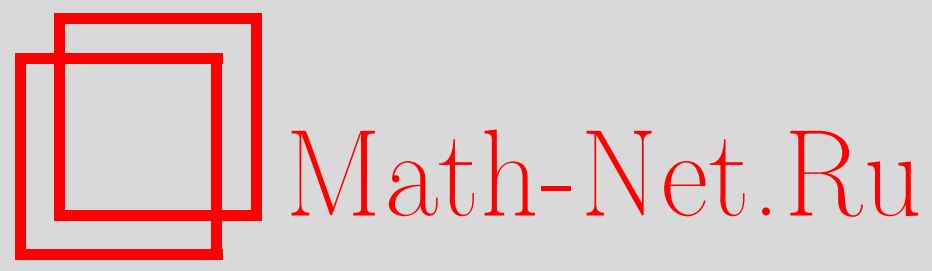

А. Н. Тимашёв, Большие уклонения для числа деревьев заданного объема и максимального объема дерева в случайном лесе, Дискрет. матем., 2006, том 18, выпуск $3,77-84$

DOI: https://doi.org/10.4213/dm60

Использование Общероссийского математического портала Math-Net.Ru подразумевает, что вы прочитали и согласны с пользовательским соглашением http://www . mathnet.ru/rus/agreement

Параметры загрузки:

IP : 54.224 .135 .184

26 апреля 2023 г., 15:10:35 
УДК 519.2

\title{
Большие уклонения для \\ числа деревьев заданного объема \\ и максимального объема дерева \\ в случайном лесе
}

(ㄷ) 2006 г. А. Н. Тимашёв

\begin{abstract}
Рассматривается множество всех лесов, состоящих из $N$ корневых деревьев, в предположении, что корни, а вместе с ними и сами деревья занумерованы числами $1, \ldots, N$, а остальные $n$ вершин каждого леса занумерованы числами $1, \ldots, n$. В предположении, что на этом множестве задано равновероятное распределение и $n, N \rightarrow \infty$, доказаны локальные предельные теоремы для распределений случайных величин, равных числу деревьев заданного объема и максимального объема дерева, позволяющие оценивать соответствующие локальные вероятности с точностью до остаточного члена известного порядка, в том числе и в области больших уклонений.
\end{abstract}

Рассмотрим класс всех однозначных отображений конечного множества в себя. Граф каждого такого отображения состоит из связных компонент с одним циклом. Если убрать дуги, соединяющие циклические вершины графа, то оставшаяся часть графа образует лес, корневыми вершинами которого являются циклические вершины. Деревья этого леса обычно называют деревьями отображения. Рассмотрим множество $F_{n, N}$ всех лесов, состоящим из $N$ корневых деревьев, в предположении, что корни, а вместе с ними и сами деревья, занумерованы числами $1, \ldots, N$, а остальные $n$ вершин каждого леса занумерованы числами $1, \ldots, n$. Если $a_{n, N}$ - число всех лесов из $F_{n, N}$, то (см. [1])

$$
a_{n, N}=N(n+N)^{n-1} .
$$

Зададим на множестве $F_{n, M}$ равномерное распределение, приписав каждому лесу из $F_{n, N}$ вероятность $a_{n, N}^{-1}$. Известно [1], что при $0<x \leqslant e^{-1}$

$$
a_{n, N}=n ! x^{-n} \exp (N \theta(x)) \mathbf{P}\left\{\xi_{1}+\ldots+\xi_{N}=n\right\}
$$

где

$$
\begin{gathered}
\theta(x)=\sum_{k=1}^{\infty} \frac{k^{k-1}}{k !} x^{k}, \\
\theta(x) e^{-\theta(x)}=x,
\end{gathered}
$$


$\xi_{1}, \ldots, \xi_{N}-$ независимые одинаково распределенные случайные величины, для которых

$$
p_{k}(x)=\mathbf{P}\left\{\xi_{1}=k\right\}=\frac{(k+1)^{k-1}}{k !} x^{k} e^{-\theta(x)}, \quad k=0,1, \ldots
$$

Как обычно, объемом дерева назовем число его некорневых вершин. Если $\mu_{r}\left(F_{n, N}\right)-$ случайная величина, равная числу деревьев объема $r$ в случайно выбранном лесе из $F_{n, N}$, To [1]

$$
\mathbf{P}\left\{\mu_{r}\left(F_{n, N}\right)=k\right\}=\left(\begin{array}{l}
N \\
k
\end{array}\right) p_{r}^{k}(x)\left(1-p_{r}(x)\right)^{N-k} \frac{\mathbf{P}\left\{\xi_{1}^{(r)}+\ldots+\xi_{N-k}^{(r)}=n-k r\right\}}{\mathbf{P}\left\{\xi_{1}+\ldots+\xi_{N}=n\right\}},
$$

где $\xi_{1}^{(r)}, \ldots, \xi_{N-k}^{(r)}-$ независимые одинаково распределенные случайные величины такие, что

$$
\mathbf{P}\left\{\xi_{1}^{(r)}=k\right\}=\mathbf{P}\left\{\xi_{1}=k \mid \xi_{1} \neq r\right\}, \quad k=0,1, \ldots, \quad k \neq r .
$$

Всюду далее, если не оговорено противное, будем предполагать, что $r$ фиксировано, $r=0,1, \ldots$

Пусть при $n, N \rightarrow \infty$ целое неотрицательное число $k=k(n, N)$ меняется так, что выполняются условия

$$
N-k \rightarrow \infty, \quad 0<\alpha_{0} \leqslant \alpha=\frac{n-k r}{N-k} \leqslant \alpha_{1}<\infty,
$$

где $\alpha_{0}, \alpha_{1}-$ постоянные и $\alpha_{0}>1$ при $r=0$.

Заметим, что если при выполнении условий (7) параметр $r \geqslant 1$, то параметры $n, N$ меняются в центральной области в том смысле, что существуют постоянные $\beta_{0}$ и $\beta_{1}$ такие, что

$$
0<\beta_{0} \leqslant \beta=n / N \leqslant \beta_{1}<\infty
$$

В случае $r=0$ условие (8) выполняется, если дополнительно предположить, что

$$
\frac{k}{N} \leqslant 1-\varepsilon
$$

где $\varepsilon-$ постоянная, $0<\varepsilon<1$.

В работе получена локальная предельная теорема, оценивающая вероятность $\mathbf{P}\left\{\mu_{r}\left(F_{n, N}\right)=k\right\}$ в предположении, что $n, N \rightarrow \infty$ и выполнены условия (7). При этом остаточный член есть $O\left((N-k)^{-1}\right)$, а параметр $k$ может попадать и в область больших уклонений. В доказательстве теоремы используются соотношения (1)-(6) и применяется метод условных распределений для сумм независимых случайных величин при специальном выборе обобщенной схемы размещения, в которой случайные величины $\xi_{1}, \ldots, \xi_{N}$ имеют одинаковое распределение вида (5) (см. [1]) Заметим, что случайные леса из множества $F_{n, N}$ изучались в работах Ю. Л. Павлова (см., например, $\left.[2,3,4]\right)$, а также других авторов (см. библ. в [4]). Однако случай, когда при $n, N \rightarrow \infty$ выполнены условия (7), до настоящего времени, по-видимому, не рассматривался, а большие уклонения для случайной величины $\mu_{r}\left(F_{n, N}\right)$ не изучались.

Наиболее близкой в идейном плане к рассматриваемому в работе кругу вопросов является статья [6], где изучаются большие уклонения высоты случайного дерева. 
Если обозначить $\eta\left(F_{n, N}\right)$ максимальный объем дерева в случайном лесе из $F_{n, N}$, то, как показано в [1], при $r=1,2, \ldots$

$$
\mathbf{P}\left\{\eta\left(F_{n, N}\right) \leqslant r\right\}=\left(\mathbf{P}\left\{\xi_{1} \leqslant r\right\}\right)^{N} \frac{\mathbf{P}\left\{\tilde{\xi}_{1}^{(r)}+\ldots+\tilde{\xi}_{N}^{(r)}=n\right\}}{\mathbf{P}\left\{\xi_{1}+\ldots+\xi_{N}=n\right\}},
$$

где $\tilde{\xi}_{1}^{(r)}, \ldots, \tilde{\xi}_{N}^{(r)}-$ независимые одинаково распределенные случайные величины такие, что

$$
\mathbf{P}\left\{\tilde{\xi}_{1}(r)=k\right\}=\frac{\mathbf{P}\left\{\xi_{1}=k\right\}}{\mathbf{P}\left\{\xi_{1} \leqslant r\right\}}, \quad k=0,1, \ldots, r .
$$

С использованием этих равенств в работе получена теорема о больших уклонениях для случайной величины $\eta\left(F_{n, N}\right)$, оценивающая вероятность $\mathbf{P}\left\{\eta\left(F_{n, N}\right) \leqslant r\right\}$ при фиксированном $r=1,2, \ldots$ и $n, N \rightarrow \infty$ в центральной области. Заметим, что случай, когда при условии (8) параметр

$$
r=\ln N\left(\ln \left(1+\beta^{-1}\right)-(1+\beta)^{-1}\right)^{-1}(1+o(1)),
$$

рассмотрен в [5].

Теорема 1. Пусть $n, N \rightarrow \infty$ и челое неотрицательное число $k=k(n, N)$ меняется так, что выполнены условия (7). Тогда равномерно относительно $\alpha \in\left[\alpha_{0}, \alpha_{1}\right]$

$$
\begin{aligned}
\mathbf{P}\left\{\mu_{r}\left(F_{n, N}\right)=k\right\}=\left(\begin{array}{l}
N \\
k
\end{array}\right) & \left(p_{r}\left(x_{r}\right)\right)^{k}\left(1-p_{r}\left(x_{r}\right)\right)^{N-k} \\
& \times \frac{n !\left(\theta\left(x_{r}\right)\right)^{N}}{N(n+N)^{n-1} x_{r}^{n+N} \sqrt{2 \pi(N-k) \sigma_{r}^{2}}}\left(1+O\left((N-k)^{-1}\right),\right.
\end{aligned}
$$

где $x_{r}-$ единственный корень уравнения

$$
\theta(x)(1-\theta(x))^{-1}=\alpha+(r-\alpha) p_{r}(x),
$$

принадлежащий интервалу $\left(0, e^{-1}\right)$, величина $p_{r}(x)$ определена в (5) $и$

$$
\begin{aligned}
\sigma_{r}^{2} & =\left(1-p_{r}\left(x_{r}\right)\right)^{-1}\left(\gamma_{r}^{3}+3 \gamma_{r}^{2}-(\alpha+r-1) \gamma_{r}+\alpha r\right)>0, \\
\gamma_{r} & =\alpha+(r-\alpha) p_{r}\left(x_{r}\right) .
\end{aligned}
$$

Доказательство. Нетрудно подсчитать, что

$$
\begin{aligned}
\mathbf{E} \xi_{i}^{(r)} & =\mathbf{E} \xi_{1}^{(r)}=\left(1-p_{r}(x)\right)^{-1}\left(\mathbf{E} \xi_{1}-r p_{r}(x)\right) \\
& =\left(1-p_{r}(x)\right)^{-1}\left(\theta(x)(1-\theta(x))^{-1}-r p_{r}(x), \quad r=0,1, \ldots, \quad i=1, \ldots, N-k .\right.
\end{aligned}
$$

Если потребовать, чтобы $\mathbf{E} \xi_{1}^{(r)}=\alpha$, то окажется, что

$$
\mathbf{E}\left(\xi_{1}^{(r)}+\ldots+\xi_{N-k}^{(r)}\right)=(N-k) \mathbf{E} \xi_{1}^{(r)}=n-k r
$$

и параметр $x$ удовлетворяет уравнению (10). Легко проверить, что при $\alpha>0$ и $r \geqslant 1$ это уравнение имеет единственный корень $x_{r} \in\left(0, e^{-1}\right)$, при $r=0$ нужно дополнительно потребовать, чтобы выполнялось неравенство $\alpha>1$. Таким образом, при условиях (7) уравнение (10) имеет ровно один корень $x_{r}$, принадлежащий интервалу $\left(0, e^{-1}\right)$. 
Вычислим дисперсию $D \xi_{1}^{(r)}$.

Нетрудно убедиться, что при $x=x_{r}$ справедливо равенство

$$
\mathbf{D} \xi_{1}^{(r)}=\sigma_{r}^{2}
$$

где $\sigma_{r}^{2}$ определяется равенствами (11), (12), при этом $\sigma_{r}^{2}>0$, поскольку $\xi_{1}^{(r)}$ имеет невырожденное распределение. Применяя к оценке $\mathbf{P}\left\{\xi_{1}^{(r)}+\ldots+\xi_{N-k}^{(r)}=n-k r\right\}$ метод перевала и учитывая, что при $x=x_{r}$

$$
\mathbf{E}\left(\xi_{1}^{(r)}+\ldots+\xi_{N-k}^{(r)}\right)=n-k r
$$

убеждаемся в том, что

$$
\mathbf{P}\left\{\xi_{1}^{(r)}+\ldots+\xi_{N-k}^{(r)}=n-k r\right\}=\frac{1}{\sqrt{2 \pi(N-k) \sigma_{r}^{2}}}\left(1+O\left((N-k)^{-1}\right)\right)
$$

равномерно относительно $\alpha \in\left[\alpha_{0}, \alpha_{1}\right]$. Заметим, что аналогичный подход использован в [7].

Полагая в (2) $x=x_{r}$ и учитывая (1), (6) и (13), получаем оценку (9). Теорема 1 доказана.

Следствие 1. Пусть $\mu_{r}\left(\Sigma_{n}^{(N)}\right)$ - число деревьев объема r в графе отображения, выбираемого случайно равновероятно в классе $\Sigma_{n}^{(N)}$ всех однозначных отображений п-элементного множества в себя, имеючих каждое ровно $N$ деревьев, и пусть при $n, N \rightarrow \infty$ целое неотричательное $k=k(n, N)$ меняется так, что $N-k \rightarrow \infty$,

$$
0<\delta_{0} \leqslant \delta=\frac{n-N-k r}{N-k} \leqslant \delta_{1}<\infty
$$

где $\delta_{0}, \delta_{1}-$ постоянные, причем $\delta_{0}>1$ nри $r=0$.

Тогда равномерно относительно $\delta \in\left[\delta_{0}, \delta_{1}\right]$

$$
\begin{aligned}
\mathbf{P}\left\{\mu_{r}\left(\Sigma_{n}^{(N)}\right)=k\right\}=\left(\begin{array}{l}
N \\
k
\end{array}\right)\left(p_{r}\left(x_{r}\right)\right)^{k}\left(1-p_{r}\left(x_{r}\right)\right)^{N-k} \\
\\
\quad \times \frac{(n-N) !\left(\theta\left(x_{r}\right)\right)^{N}}{N n^{n-N-1} x_{r}^{n} \sqrt{2 \pi(N-k) \sigma_{r}^{2}}}\left(1+O\left((N-k)^{-1}\right)\right),
\end{aligned}
$$

где параметры $x_{r}$ и $\sigma_{r}^{2}$ определяются согласно (10)-(12) с заменой $\alpha$ на $\delta$.

Замечание 1. Выбор параметра $x=x_{r}$ в процессе доказательства теоремы 1 обусловлен тем обстоятельством, что при другом значении $x$ остаточный член в (13) будет иметь порядок $O\left((N-k)^{-1 / 2}\right)$, что увеличивает относительную погрешность при использовании оценки, аналогичной (9).

Замечание 2. Теорема 1 позволяет оценить вероятность $\mathbf{P}\left\{\mu_{r}\left(F_{n, N}\right)=k\right\}$ в области больших уклонений случайной величины $\mu_{r}\left(F_{n, N}\right)$. Исключением является случай, когда при условиях этой теоремы

$$
k=N p_{r}\left(x_{r}\right)+y \sqrt{N}, \quad|y| \leqslant C,
$$


где $C$ - положительная постоянная. В этом случае можно показать, что равномерно относительно $\alpha \in\left[\alpha_{0}, \alpha_{1}\right]$ и целых положительных $k$

$$
\begin{aligned}
& \mathbf{E} \mu_{r}\left(F_{n, N}\right)=N p_{r}\left(x_{r}\right)\left(1+O\left(N^{-1 / 2}\right)\right), \\
& \mathbf{D} \mu_{r}\left(F_{n, N}\right)=N\left(\sigma^{(r)}\right)^{2}\left(1+O\left(N^{-1 / 2}\right),\right.
\end{aligned}
$$

где

$$
\begin{aligned}
\left(\sigma^{(r)}\right)^{2} & =\frac{\sigma_{r}^{2}}{\sigma^{2}}\left(1-p_{r}\left(x_{r}\right)\right)^{2} p_{r}\left(x_{r}\right)>0, \\
\sigma^{2} & =\theta\left(x_{r}\right)\left(1-\theta\left(x_{r}\right)\right)^{-3}>0 .
\end{aligned}
$$

Кроме того, в этом случае

$$
\begin{aligned}
\mathbf{P}\left\{\mu_{r}\left(F_{n, N}\right)=k\right\}= & \frac{1}{\sqrt{2 \pi N\left(\sigma^{(r)}\right)^{2}}} \\
& \times \exp \left(-\frac{y^{2}}{2}\left(\frac{1}{p_{r}\left(x_{r}\right)\left(1-p_{r}\left(x_{r}\right)\right)}-\frac{(r-\alpha)^{2}}{\sigma^{2}}\right)\right)\left(1+O\left(N^{-1 / 2}\right)\right)
\end{aligned}
$$

равномерно относительно $\alpha \in\left[\alpha_{0}, \alpha_{1}\right]$ и целых положительных

$$
k=N p_{r}\left(x_{r}\right)+y \sqrt{N}, \quad|y| \leqslant C,
$$

где $C$ - положительная постоянная. Последний результат, как нетрудно проверить, является частным случаем доказанной Ю. Л. Павловым в [2] локальной нормальной теоремы для случайной величины $\mu_{r}\left(F_{n, N}\right)$.

Замечание 3. Чтобы решить уравнение (10) с любой заданной степенью точности, следует положить

$$
\theta(x)=t, \quad \theta\left(x_{r}\right)=t_{r}, \quad r=0,1, \ldots
$$

В этих обозначениях согласно (10) и условиям теоремы $1 t=t_{r}-$ единственный корень уравнения

$$
\frac{t}{1-t}=\alpha+\frac{(r-\alpha)(r+1)^{r-1}}{r !} t^{r} e^{-(r+1) t},
$$

принадлежащий интервалу $(0,1)$. Найдя из $(15)$ значение $t_{r}$ стандартными методами, далее используем соотношение

$$
x_{r}=t_{r} e^{-t_{r}}
$$

Если учесть, что согласно (10), (12)

$$
\gamma_{r}=t_{r}\left(1-t_{r}\right)^{-1}, \quad r=0,1, \ldots,
$$

то можно вычислить дисперсию $\sigma_{r}^{2}$ в (11), а также дисперсии $\sigma^{2}=t_{r}\left(1-t_{r}\right)^{-3}$ и $\left(\sigma^{(r)}\right)^{2}$.

Так, например, если $n=10, N=8, r=0$, то $t_{0}=0,141899 \ldots$ с точностью до $10^{-6}$, и оценка вероятности $\mathbf{P}\left\{\mu_{0}\left(F_{10,8}=0\right\}\right.$ по формулам (9)-(12) при $k=0$ дает величину $2,0110 \cdot 10^{-4}$ вместо значения $1,9285 \cdot 10^{-4}$ (все 5 знаков верны).

Если же $n=10, r=0, N=60, k=52$, то оценка вероятности $\mathbf{P}\left\{\mu_{0}\left(F_{10,60}\right)=52\right\}$ дает величину 0,3372 вместо 0,3234 (с точностью до $10^{-4}$ ). В то же время использование локальной нормальной теоремы (см. замечание 2) в хорошем случае, когда $y$ достаточно мало (при наших условиях $y$ принимает значение близкое к -0,008) дает оценку 0,3388 (с той же точностью). 
Замечание 4. Используя метод моментов, нетрудно убедиться в том, что если $n, N \rightarrow \infty$ так, что

$$
N\left(\frac{n}{N}\right)^{r} \rightarrow \frac{\lambda r !}{(r+1)^{r-1}}
$$

где $\lambda>0$, то случайная величина $\mu_{r}\left(F_{n, N}\right)$ распределена в пределе по закону Пуассона с параметром $\lambda, r=2,3, \ldots$ Такое же заключение справедливо, если $n, N, r \rightarrow \infty$ так, что $r=o(\sqrt{n})$ и

$$
N\left(\frac{n}{n+N}\right)^{r} \exp \left(\frac{(r+1) N}{n+N}\right) r^{-3 / 2} \rightarrow \lambda \sqrt{2 \pi}
$$

где $\lambda>0$.

Оба сформулированных результата, как несложно проверить, являются следствиями предельной теоремы пуассоновского типа, доказанной Ю. Л. Павловым в [2].

Теорема 2. Пусть $\eta\left(F_{n, N}\right)$ - максимальный объем дерева в случайном лесе из $F_{n, N}, u$ пусть $n, N \rightarrow \infty$ так, что выполнено условие (8), причем

$$
\beta_{1}<e^{-1} S_{r}^{\prime}\left(e^{-1}\right)\left(S_{r}\left(e^{-1}\right)\right)^{-1} \text {. }
$$

Тогда равномерно относительно $\beta_{1} \in\left[\beta_{0}, \beta_{1}\right]$

$$
\mathbf{P}\left\{\eta\left(F_{n, N}\right) \leqslant r\right\}=\frac{1}{\sqrt{2 \pi N\left(\sigma_{r}^{*}\right)^{2}}} \frac{n !\left(S_{r}\left(x_{r}^{*}\right)\right)^{N}}{N(n+N)^{n-1}\left(x_{r}^{*}\right)^{n}}\left(1+O\left(N^{-1}\right)\right),
$$

где $x_{r}^{*}$ - единственный корень уравнения

$$
x S_{r}^{\prime}(x)\left(S_{r}(x)\right)^{-1}=\beta,
$$

принадлежащий интервалу $\left(0, e^{-1}\right)$,

$$
\begin{aligned}
& S_{r}(x)=\sum_{m=0}^{r} \frac{(m+1)^{m-1}}{m !} x^{m}, \\
& \left(\sigma_{r}^{*}\right)^{2}=\left(x_{r}^{*}\right)^{2} S_{r}^{\prime \prime}\left(x_{r}^{*}\right)\left(S_{r}\left(x_{r}^{*}\right)\right)^{-1}+\beta(1-\beta)>0 .
\end{aligned}
$$

Доказательство. Используем для вероятности $\mathbf{P}\left\{\eta\left(F_{n, N}\right) \leqslant r\right\}$ приведенную выше формулу, полагая $x=x_{r}^{*}$. Поскольку функция

$$
f(x)=x S_{r}^{\prime}(x)\left(S_{r}(x)\right)^{-1}
$$

непрерывна и строго возрастает на интервале $\left(0, e^{-1}\right)$ от 0 до значения $f\left(e^{-1}\right)$, согласно $(8),(16)$ уравнение (18) имеет единственный корень $x_{r}^{*} \in\left(0, e^{-1}\right)$. При этом согласно (5) и (19) при $x=x_{r}^{*}$

$$
\mathbf{P}\left\{\xi_{1} \leqslant r\right\}=e^{-\theta\left(x_{r}^{*}\right)} S_{x}\left(x_{r}^{*}\right),
$$

и при $k=0,1, \ldots, r$

$$
\mathbf{P}\left\{\tilde{\xi}_{1}^{(r)}=k\right\}=\frac{(k+1)^{k-1}}{k !}\left(x_{r}^{*}\right)^{k}\left(S_{r}\left(x_{r}^{*}\right)\right)^{-1}
$$


Поэтому

$$
\begin{aligned}
\mathbf{E} \tilde{\xi}_{1}^{(r)}=x_{r}^{*} S_{r}^{\prime}\left(x_{r}^{*}\right)\left(S_{r}\left(x_{t}^{*}\right)\right)^{-1}=\beta, \\
\mathbf{E}\left(\tilde{\xi}_{1}^{(r)}+\ldots+\tilde{\xi}_{N}^{(r)}\right)=n .
\end{aligned}
$$

Используя метод перевала, получаем, что равномерно относительно $\beta \in\left[\beta_{0}, \beta_{1}\right]$

$$
\mathbf{P}\left\{\tilde{\xi}_{1}^{(r)}+\ldots+\tilde{\xi}_{N}^{(r)}=n\right\} \frac{1}{\sqrt{2 \pi N\left(\sigma_{r}^{*}\right)^{2}}}\left(1+O\left(N^{-1}\right)\right)
$$

где, как легко проверить, $\mathbf{D} \tilde{\xi}_{1}^{(r)}=\left(\sigma_{r}^{*}\right)^{2}$ и значение $\left(\sigma_{r}^{*}\right)^{2}$ определяется равенством (20).

Далее, при $x=x_{r}^{*}$ из (1), (2) следует, что

$$
\mathbf{P}\left\{\xi_{1}+\ldots+\xi_{N}=n\right\}=\frac{N(n+N)^{n-1}\left(x_{r}^{*}\right)^{n}}{n !} \exp \left(-N \theta\left(x_{r}^{*}\right)\right)
$$

Подставляя значения вероятностей из (21)-(23) в указанную выше формулу, получаем оценку (17).

Теорема 2 доказана.

В практических расчетах оценка (17) оказывается весьма точной даже при небольших значениях $n, N$. Если взять, например, $r=2, n=6, N=18$, то формула (17) дает оценку 0,6508 вместо значения $\mathbf{P}\left\{\eta\left(F_{6,18}\right) \leqslant 2\right\}=0,6374 \ldots$ В то же время использование соответствующей оценки из [5] при указанных условиях дает значение 0,4081 .

Следствие 2. Пусть $\eta\left(\Sigma_{n}^{(N)}\right)$ - максимальный объем дерева в графе отображения, выбираемого так же, как в следствии 1 , и пусть $n, N \rightarrow \infty$ так, что

$$
1<\beta_{0} \leqslant \beta=n / N \leqslant \beta_{1}<1+e^{-1} S_{r}^{\prime}\left(e^{-1}\right)\left(S_{r}\left(e^{-1}\right)\right)^{-1}
$$

где $\beta_{0}, \beta_{1}$ - постоянные. Тогда равномерно относительно $\beta \in\left[\beta_{0}, \beta_{1}\right]$

$$
\begin{aligned}
\mathbf{P}\left\{\eta\left(\Sigma_{n}^{(N)} \leqslant r\right\}=\frac{1}{\sqrt{2 \pi N\left(\sigma_{r}^{*}\right)^{2}}}(n\right. & -N) ! \\
& \times\left(S_{r}\left(x_{r}^{*}\right)\right)^{N} N^{-1} n^{N+1-n}\left(x_{r}^{*}\right)^{N-n}\left(1+O\left(N^{-1}\right)\right),
\end{aligned}
$$

где $r=1,2, \ldots$, параметры $x_{r}^{*} u\left(\sigma_{r}^{*}\right)^{2}$ определяются из (18), (20) с заменой $\beta$ на $\beta-1$.

Оценка (24) следует из (17) с учетом соотношения из [1]

$$
\mathbf{P}\left\{\eta\left(\Sigma_{n}^{(N)}\right) \leqslant r\right\}=\mathbf{P}\left\{\eta\left(F_{n-N, N}\right) \leqslant r\right\}, \quad r=1,2, \ldots
$$




\section{Список литературы}

1. Колчин В. Ф., Случайные отображения. Наука, Москва, 1984.

2. Павлов Ю. Л., Предельные теоремы для числа деревьев заданного объема в случайном лесе. Матем. сб. (1977) 103, №3, 392-403.

3. Павлов Ю. Л., Случайные леса. Карельский научн. центр, Петрозаводск, 1996.

4. Pavlov Yu. L., Random forests. In: Probab. Methods in Discrete Math. VSP, Utrecht, 1997, pp. 11-18.

5. Павлов Ю. Л., Асимптотическое распределение максимального объема дерева в случайном лесе. Теория вероятностей и ее применения (1977) 22, №3, 523-533.

6. Макаров Г. Д., Большие уклонения высоты случайного дерева. Дискретная математика (1993) 5, №2, 116-125.

7. Тимашев А. Н., Случайные разбиения множеств с известным числом блоков. Дискретная математика (2003) 15, №2, 138-148.

Статья поступила 09.06.2004. 